\title{
Protective experience of diabetic patients with 2019 novel coronavirus-infected pneumonia (2019-nCoV) in Wuhan, China
}

\section{Ma Lihua}

the first hospital of Lanzhou University https://orcid.org/0000-0002-1735-6631

Cheng Jianguo

The first hospital of Lanzhou University

\section{Zhuang Kaipeng}

The 940 Hospital of Joint Logistic Support force of Chinese People's Liberation Army

\section{Li Juan}

The first hospital of Lanzhou University

\section{Zhang Tao ( $\sim$ jhshuoshi@163.com )}

https://orcid.org/0000-0001-6592-8086

\section{Research article}

Keywords: 2019 novel coronavirus-infected pneumonia, diabetes, protective and nursing

Posted Date: March 19th, 2020

DOl: https://doi.org/10.21203/rs.3.rs-18038/v1

License: (9) This work is licensed under a Creative Commons Attribution 4.0 International License. Read Full License 


\section{Abstract}

\section{Background}

Since December 2019, more than 77,665 patients have been diagnosed with 2019 novel coronavirusinfected pneumonia (NCIP) in Wuhan, Hubei Province. According to previously published data, most of the critical and deceased patients are middle-aged and elderly patients with complications related to multiple chronic diseases. Diabetes is one of the most common chronic diseases. On January 29, The Lancet reported that 41 patients were infected by $2019-\mathrm{nCoV}$, and 8 patients were complicated with diabetes, accounting for $20 \% 1$. It has been suggested that diabetic patients are likely more susceptible to 2019-nCoV.At present, there is no effective treatment method or vaccine for NCIP. The prognosis of patients mainly depends on their immune system. However, diabetic patients with NCIP have higher requirements for treatment and nursing due to their complicated condition. To summarize the protective experience and provide a basis for hospital and patient care, we reviewed the protective mechanisms and effects in 12 diabetic patients with 2019 novel coronavirus-infected pneumonia (2019-nCoV) in Wuhan, China.

\section{Methods}

During nursing care, strict isolation and protection were emphasized, which included in-hospital isolation and protection, ward disinfection and isolation, ward environment layout, self-protection of medical staff, specimen collection and transportation; disease condition monitoring included blood glucose management, nutrition support, basic nursing, medication care, psychological support and discharge instructions. One patient died of complications due to gastrointestinal haemorrhage, and the other 11 patients were cured and discharged.

Discussion

Strict isolation, protection and disease monitoring are prerequisites for reducing complications and improving the cure rate of 2019-nCoV.

\section{Background}

Since December 2019, more than 77,665 patients have been diagnosed with 2019 novel coronavirusinfected pneumonia (NCIP) in Wuhan, Hubei Province. According to previously published data, most of the critical and deceased patients are middle-aged and elderly patients with complications related to multiple chronic diseases. Diabetes is one of the most common chronic diseases. On January 29, The Lancet reported that 41 patients were infected by $2019-n C o V$, and 8 patients were complicated with diabetes, accounting for $20 \%$ [1]. It has been suggested that diabetic patients are likely more susceptible to 2019-nCoV. 
In 2003, the mortality rate of diabetic patients with acute respiratory distress syndrome (SARS), the need for mechanical ventilation treatment, and the rate of intensive care unit (ICU) admissions were 3.1 times higher than those of non-diabetic patients [2]. In 2009, the number of diabetic patients admitted to the ICU for treatment for influenza A (H1N1) was 4.29 times that of non-diabetic patients [3]. Diabetes was also a high-risk factor for an outbreak of Middle East Respiratory Syndrome coronavirus (MERS-CoV) among critical care patients in 2014 [4]. Diabetic patients often have abnormal immune functions such as decreased CD3 $+T$ cells, an imbalance of CD4+/CD8 + T cells, decreased NKT cell activity, and decreased immune response ability. Diabetic patients comprise a high-risk group for virus infection [3]. However, diabetes may be further aggravated after virus infection. Angiotensin converting enzyme 2 (ACE2), a functional receptor of SARS-CoV, is also expressed in the islets of Langerhans. During patient infection, the virus may destroy the islets of Langerhans through this receptor, aggravating diabetes and exacerbating disease progression [5]. Recent studies in China have found that 2019-nCoV can also effectively use ACE2 to enter cells and infect human beings [6]. The viral infection interacts with diabetes, making patients with 2019-nCoV more likely to develop critical infections leading to death.

At present, there is no effective treatment method or vaccine for NCIP. The prognosis of patients mainly depends on their immune system. However, diabetic patients with NCIP have higher requirements for treatment and nursing due to their complicated condition. The nursing experience of 12 diabetic patients with NCIP is described as follows.

\section{Objective}

To summarize the protective experience and provide a basis for hospital and patient care, we reviewed the protective mechanisms and effects in 12 diabetic patients with 2019 novel coronavirus-infected pneumonia (2019-nCoV) in Wuhan, China.

\section{Methods}

\section{Participants}

The patients were enrolled from the affiliated hospital of Tongji Medical College of Huazhong University of Science and Technology, Zhongnan Hospital of Wuhan University, Pu'ai Hospital Affiliated to Huazhong University of Science and Technology Wuhan Fourth Hospital and Fangcang Hospital. From January 29, 2020 to February 20,2020, the members of our research group cared for 12 patients with diabetes complicated with NCIP, aged from 46 to 78 years old, including 7 males and 5 females, 2 patients with type 1 diabetes and 10 patients with type 2 diabetes.

\section{Clinical Manifestations}


Twelve diabetic patients with pneumonia caused by 2019-nCoV had basic symptoms of pneumonia caused by 2019-nCoV, such as fever, dry cough, chest tightness, shortness of breath, dyspnoea, and muscle soreness, as well as diabetes symptoms, such as fatigue, hunger, and thirst. Among these patients, one patient used hormones, which caused a stress ulcer in the digestive tract to haemorrhage, and one patient had multiple pressure sores upon admission (area of large pressure ulcers: $5 \mathrm{~cm} \star 3 \mathrm{~cm}$, area of small pressure ulcers: $1 \mathrm{~cm} * 0.5 \mathrm{~cm}$ ).

\section{Ethics Statement}

Ethical approval was obtained from ethics review committees at the First Hospital of Lanzhou University (Lanzhou, China) prior to the survey launch. Inform consent was obtained from all the patients by esigning the online informed consent.

\section{Results}

After more than 20 days of treatment and nursing, 11 diabetic patients with NCIP were cured and discharged from the hospital. One patient died of gastrointestinal haemorrhage. One patient with pressure ulcers had a normal temperature for 3 days, their respiratory symptoms improved significantly, and respiratory pathogenic nucleic acid detection was negative 2 consecutive times (sampling interval 1 day); therefore, in-hospital infection was prevented. The patient's wound improved, and the patient was discharged from the hospital.

\section{Discussion}

\section{In-hospital protection}

Strict in-hospital isolation and protection are urgently needed: NCIP is spread in crowds through droplets, contact, aerosols and the digestive tract. This epidemic mainly occurs in large cities, where the population is relatively concentrated, and spreads rapidly, thus causing an NCIP outbreak. Strict control of in-hospital infection is vital to control the further spread of the epidemic. Based on the crowded situation at clinics described in various news reports, in-hospital infection may be the main driving factor of the outbreak. Clinical experts from Beijing visited the clinics without goggles and claimed to have contracted 2019nCoV. As of January 26, the mayor of Wuhan announced that less than $50 \%$ of undiagnosed patients in hospitals, including clinics, were 2019-nCoV infections. How many people were cross-infected in hospitals when a large number of non-infected patients visited the hospital clinics? Although the author does not have any data to determine this number at present, many models now estimate that the basic reproduction number of 2019-nCoV transmission in the population is between 2 and 6.5, which indicates that the disease is highly infectious [7-10]. However, the basic number of regenerations spread by the disease in patient-intensive hospitals and general communities may be fundamentally different. This is also one of the reasons why many researchers are pessimistic about the prediction of epidemic trends of 
diseases. Therefore, the main focus of prevention and control in various locations at present is to attach great importance to cross-infection in hospitals, including but not limited to clinics, and nosocomial infection in hospitals is a key link to be controlled. Diabetes mellitus patients have low immunity and can easily be infected due to their high blood sugar level, which aggravates the illness after infection with 2019-nCoV. A systematic review suggested that aerosol-producing operations (such as tracheal intubation, tracheotomy, mechanical ventilation, and fibreoptic bronchoscopy) increase the risk of SARSCoV and MERS-CoV transmission [11]. Therefore, strict isolation and protection are important measures throughout the hospitalization period of patients.

\section{Self-protection of Medical Staff}

After one patient was diagnosed with NCIP, we strictly arranged the patients in a single ICU ward for isolation in accordance with the technical guidelines for prevention and control of NCIP in medical institutions issued by the Medical Administration and Hospital Authority of the National Health Council. Meanwhile, medical staff wear N95 masks, protective clothing, goggles and double gloves [12]. Doctors wear disposable hoods for tracheal intubation and extubation of patients and use closed sputum suction tubes for sputum aspiration of patients with disposable protective face screens. Hand hygiene must be carried out at the proper time (before and after contacting the patient, after contacting the bed unit of the patient and the surrounding environment). The surface and floor of the ward must be wiped twice a day with $2000 \mathrm{mg} / \mathrm{L}$ chlorine-containing disinfectant. The medical air disinfection device must be recycled for 24 hours. The window was opened and ventilated at least twice a day for more than half an hour each time. Medical waste was treated strictly in accordance with the management requirements of Class $\mathrm{A}$ infectious diseases. The patients stayed in the ICU for 20 days, and none of the medical staff members developed infection.

\section{Ward environment protection}

Structure and Layout of the Ward Environment: 1) Area separation: The contaminated area, semicontaminated area and clean area of the ward must be strictly separated, and the three areas are divided into distinct zones, without cross use. 2) Possessions of NCIP patients: All possessions of NCIP patients should be located in a centralized area. If conditions permit, they should be isolated in a single room with a bed spacing of not less than 1.2 metres. If a transmission window is present in the ward, it should be irradiated and disinfected with ultraviolet light for 30 minutes/time twice a day. 3) Air disinfection: Curtains should be removed, and ventilation should be increased. The air disinfection unit should be run for 24 hours a day. Wards with laminar flow should regularly perform filter screens according to laminar flow specifications. A hydrogen peroxide space sterilizer should be used for final disinfection of deceased patients. Hydrogen peroxide (3\%), $5000 \mathrm{mg} / \mathrm{L}$ peracetic acid or $500 \mathrm{mg} / \mathrm{L}$ chlorine dioxide should be used in an ultra-low volume atomizer for spray disinfection (20-30 $\mathrm{ml} / \mathrm{m}^{3}$ for 2 hours). Doors and windows should be closed during disinfection and can only be used after strict disinfection and full ventilation for at least 1 hour. 4) Daily articles: Daily articles should be wiped with paper towels containing peracetic acid twice a day and recorded. A special cleaning tool should be used for the specified area. For patient excrement, secretions, vomit and other contaminated substances, we used absorbent materials such as 
paper towels to remove visible contaminants. Then, disinfectant containing $1000 \mathrm{mg} / \mathrm{L}$ chlorine was used to wipe for 30 minutes, and then further wiping and disinfection were performed. Bed sheets, quilt covers, and clothes were sealed in orange bags. The bags were marked with "2019-nCoV ", the patient's name and quantity of the items was recorded, and the bags were sent to the laundry room. 5) Thermometer: Thermometers were soaked in $1000 \mathrm{mg} / \mathrm{L}$ Jianzhisu for 30 minutes after each use and then washed and dried for later use. Ventilator tubes should be soaked with $2000 \mathrm{mg} / \mathrm{L}$ Jianzhisu for 30 minutes and then sealed with double-layer yellow plastic bags marked with "2019-nCoV" and sent to the disinfection supply centre for processing with a handover record. 6) Disposable articles: Disposable articles such as tongue depressors, tourniquets, and sputum suction tubes should be used. Resuscitation bags should be specifically assigned to patients.7) Medical care documents: Medical care documents were signed by the family members of the patient and could only be taken into the ward for filing after being placed in the transmission window and irradiated by an ultraviolet lamp for 30 minutes. 8) Terminal disinfection: After disinfection, all surfaces were wiped with $2000 \mathrm{mg} / \mathrm{L}$ chlorine-containing disinfectant. The ventilator should not be used for one week after disinfection. After a patient death, the body should be treated in a timely manner. The treatment method consists of using cotton balls or gauze with $3000 \mathrm{mg} / \mathrm{L}$ of Jianzhisu to fill all open channels of the patient such as the mouth, nose, ears and anus. The corpse should be wrapped with a double-layer cloth sheet, then wrapped with a sealed anti-leak double-layer corpse bag, and immediately sent to the funeral home for cremation (see to Table 1). 
Table 1

Recommendations for disinfection of objects contaminated with 2019-nCoV

\begin{tabular}{|c|c|c|}
\hline $\begin{array}{l}\text { Contaminated } \\
\text { object }\end{array}$ & Disinfectant & Type of disinfection \\
\hline Indoor air & $\begin{array}{l}\text { Peracetic acid, } \\
\text { hydrogen } \\
\text { peroxide, etc. }\end{array}$ & Spray method \\
\hline $\begin{array}{l}\text { Contaminants } \\
\text { (patient } \\
\text { blood, } \\
\text { secretions, } \\
\text { etc.) }\end{array}$ & $\begin{array}{l}\text { Chlorine } \\
\text { disinfectant } \\
(5000 \mathrm{mg} / \mathrm{L}- \\
10,000 \mathrm{mg} / \mathrm{L})\end{array}$ & $\begin{array}{l}\text { A small amount can be used as a dipping disinfectant for } \\
\text { disposable absorbent material }\end{array}$ \\
\hline $\begin{array}{l}\text { Floor, wall, } \\
\text { elevator }\end{array}$ & $\begin{array}{l}\text { Chlorine } \\
\text { disinfectant } \\
\text { (1000 mg/L) or } \\
\text { chlorine } \\
\text { dioxide } \\
(500 \mathrm{mg} / \mathrm{L})\end{array}$ & Wipe or spray \\
\hline $\begin{array}{l}\text { Object } \\
\text { surfaces }\end{array}$ & $\begin{array}{l}\text { Chlorine } \\
\text { disinfectant } \\
\text { ( } 1000 \mathrm{mg} / \mathrm{L}) \text { or } \\
\text { chlorine } \\
\text { dioxide } \\
(500 \mathrm{mg} / \mathrm{L})\end{array}$ & Wipe, spray or soap disinfection \\
\hline $\begin{array}{l}\text { Bedding and } \\
\text { other fabrics }\end{array}$ & $\begin{array}{l}\text { Chlorine } \\
\text { disinfectant } \\
\text { ( } 500 \mathrm{mg} / \mathrm{L})\end{array}$ & $\begin{array}{l}\text { It is recommended that all incineration disposal should be carried } \\
\text { out as medical waste; if an item needs to be reused by the } \\
\text { patient, it should first be immersed and sterilized with } 500 \mathrm{mg} / \mathrm{L} \\
\text { chlorine-containing disinfectant and then cleaned as usual }\end{array}$ \\
\hline
\end{tabular}

\section{Specimen collection and transportation}

Gargle samples should be collected at the early stage of the disease (preferably within 5 days) to improve the virus detection rate. The collection time should be during fasting or 2 hours after eating, while drinking as little water as possible during that time. During sampling, $5 \mathrm{ml}$ of sterile physiological saline should be collected in a $15 \mathrm{ml}$ standard, sealed, disposable, sterile, plastic centrifuge tube with a cover. The patient should first cough twice and then gargle with $5 \mathrm{ml}$ of sterile physiological saline. When gargling, the patient's head should be tilted back while making an "ah" sound, so that the sampling solution is transferred to the pharynx for $3-5$ seconds and then slowly expectorated into the centrifuge tube through a paper funnel.

Excrement collection should be carried out using sterilized bamboo sticks. Excrement containing pus, blood or mucus should be placed in a $15 \mathrm{ml}$ standard sealed disposable sterile plastic centrifuge tube with a cover. Rinse samples and stool samples should be transported on ice (pre-prepared) and sent to the laboratory as soon as possible.

\section{Family visitation management}


Management of patient family members: Family members of patients are an important floating population in the hospital, and their awareness and infection prevention ability are low. Temporary family visitation restrictions can reduce the risk of cross-infection in hospitals by reducing hospital flow and exposure opportunities during the epidemic period of 2019-nCoV [13]; these procedures have been widely adopted during the epidemic periods of various infectious diseases $[14,15]$. However, improper use of temporary family visitation restrictions may lead to negative psychosocial impact, interruption of communication, decline in the care level and other risks [13]. This requires hospitals to assess the risk of psychosomatic damage to patients according to their actual situation and formulate countermeasures to protect patients to the greatest extent possible when formulating a visitor restriction system. Although qualitative research results suggest that patients had a good understanding of visiting restrictions during the SARS epidemic, the opinions of patients and their families should be noted to prevent a decline of the patient's medical experience and potential conflicts between doctors and patients [16].

According to our experience and previous experience reported in the literature, face-to-face visits of NCIP patients in the ICU and general wards should be considered (family members with a contact history with patients should actively seek medical observation), visits and accompanying patients with non-NCIP in general wards should be limited (the number of family members should not exceed 1 person per day), and appropriate personnel should be present to ensure strict implementation of the system. To avoid loneliness in patients and meet their communication needs, communication forms such as remote voice or video visits can be considered [17].

\section{Patient care}

Blood sugar management: when diabetic patients were infected with NCIP, the body was in a state of stress. This study showed that the fasting pre-hospital blood glucose level of patients was 11.7$13.9 \mathrm{mmol} / \mathrm{L}$, and the postprandial blood glucose level was $14.1-19.3 \mathrm{mmol} / \mathrm{L}$. During anti-inflammatory therapy, patients may require a large amount of adrenocortical hormone therapy because adrenocortical hormone is the anti-regulatory hormone of insulin, which will cause the blood sugar level to increase. In this study, patients had high fasting blood glucose levels that reached $20.4 \mathrm{mmol} / \mathrm{L}$.

Increasing blood sugar monitoring: Increasing blood sugar monitoring and controlling hyperglycaemia are the key links in the treatment and nursing of diabetes patients complicated with NCIP. Specific methods: The insulin dose should be increased appropriately according to the doctor's orders, and a fingertip blood glucose meter should be used to monitor the blood glucose of patients 7 times a day (before the 3 meals, 2 hours after the 3 meals and before sleep); the blood glucose level should be monitored at night when necessary in critically ill patients. Insulin levels should be adjusted in a timely manner to prevent hyperglycaemic crises, such as diabetic ketoacidosis or hypertonic coma caused by insulin deficiency and hypoglycaemia caused by insulin overdose, and to discover hypoglycaemic signs in patients as early as possible. The blood glucose management targets of hospitalized diabetic patients are listed in Table 2 (to see Table 2) [18]. The control targets are strict for young, newly diagnosed or short-course diabetic patients and the low-risk population with hypoglycaemia. Old age, intolerance of hypoglycaemia, organ dysfunction or serious cardiovascular and cerebrovascular diseases, loose or 
general control objectives, severe or critical cases of new coronavirus pneumonia: loose control objectives.

Table 2

Target Stratification of Blood Glucose Management for Inpatients with Diabetes

\begin{tabular}{|llll|}
\hline & Strict & General & Loose \\
\hline Fasting/preprandial blood glucose (mmol/L) & $4.4-6.1$ & $6.1-7.8$ & $7.8-10.0$ \\
\hline 2 h after meal or random blood sugar (mmol/L) & $6.1-7.8$ & $7.8-10.0$ & $7.8-13.9$ \\
\hline $\begin{array}{l}\text { If the patient has a high risk of hypoglycaemia, individualized control targets are recommended } \\
\text { according to clinical conditions and complications. }\end{array}$ & & \\
\hline $\begin{array}{l}\text { * High-risk group for hypoglycaemia: patients with a diabetic course } \geq 15 \text { years, no history of } \\
\text { perceived hypoglycaemia, large fluctuation of blood sugar throughout the day with recurrent } \\
\text { hypoglycaemia, severe complications or concomitant diseases such as hepatic and renal } \\
\text { insufficiency. }\end{array}$ & & \\
\hline
\end{tabular}

Observation of complications: Diabetic patients often experience complications involving the functions of various organs and structural changes, resulting in multiple organ failure and death after infection with NCIP. Therefore, we monitored the vital signs of a patient with gastrointestinal haemorrhage according to the patient's condition, observed whether signs of haemorrhage were present, recorded 24-hour intake and discharge, and paid attention to the examination results of haemoglobin, haematochezia, and occult blood.

\section{Concomitant hypoglycaemic coma}

One patient had hypoglycaemia $(3.1 \mathrm{mmol} / \mathrm{L})$ at night, and all hypoglycaemic drugs were stopped immediately. Intravenous injection of $60 \mathrm{ml}$ of $50 \%$ glucose solution followed by continuous intravenous infusion of $5-10 \%$ glucose were administered until consciousness was restored and maintained. The blood sugar level was monitored for at least 3 days, and a safer hypoglycaemic strategy was selected.

\section{Increased nutritional support}

For patients with type 2 diabetes, regular diet is a basic treatment method and an important way to control blood sugar and relieve symptoms. However, during the treatment of NCIP, after using a large amount of hormone therapy, the blood sugar stress increases. In addition, a high fever increases energy use and the feeling of hunger in patients; therefore, it is difficult to control the diet. The four nutritionists provided special dietary guidance. On the one hand, the diet structure was reasonably adjusted to ensure the supply of calories; on the other hand, the patients were supervised to eat on time. Additionally, in 4 patients with a $\mathrm{BMI}<18.5 \mathrm{~kg} / \mathrm{m}^{2}$ dietary restrictions were relaxed, while insulin was used to control the blood sugar, thus improving the nutritional status of the patients and increasing resistance. Three patients with critical illnesses who were unable to eat normally required injection of liquid to ensure basic energy, and the insulin dose was adjusted according to the input and detection of glucose. 


\section{Primary care}

Nursing care of patients with high fever: Attention should be paid to cooling: During cold application, application of ice on the same part for a long time should be avoided to prevent local frostbite. Attention should be paid to the peripheral circulation, especially in patients with a fine pulse rate, pale complexion and cold limbs, and cold compresses and alcohol should not be used. Patients with a bleeding tendency should not be placed in an alcohol bath. When applying drugs to reduce temperature, care should be taken not to excessively lower the body temperature in a short period of time to avoid collapse caused by sweating.

Pressure ulcer care: Intensive basic care: Due to hyperglycaemia in patients, coupled with the effect of high fever and sweating, skin resistance decreases, and sensitivity to pressure and various stimuli increases. Meanwhile, neutrophil and phagocyte functions are disrupted, and transfer factors fail to increase correspondingly to promote wound healing. Once the damage occurs, infection can easily occur, and wounds become difficult to heal. Therefore, special attention should be paid to increasing basic nursing care for diabetes patients complicated with NCIP. In the nursing process, effective nursing measures should be taken according to the NCIP characteristics, symptoms, and illness changes. For 13 NCIP patients with dyspnoea symptoms such as high fever, chest tightness, and shortness of breath, physical cooling and forced posture were required. We focused on keeping the skin clean and dry, removing dandruff in a timely manner, providing morning and evening care for patients and changing clothes in a timely manner. For a patient with NCIP accompanied by gastrointestinal haemorrhage who required absolute bed rest due to his illness, an air cushion bed was used to encourage and assist the patient to turn over regularly and change body positions to prevent skin injury during turning over. Meanwhile, foot movement is performed to improve blood circulation of the lower limbs and prevent thrombosis. After haematochezia, the anal circumference should be kept clean and dry. Another patient was admitted to the hospital with 3 pressure sores on the sacrococcygeal region, hip and heel, which were $5 \mathrm{~cm} * 3 \mathrm{~cm}$ (phase II), $2 \mathrm{~cm} * 1 \mathrm{~cm}$ (phase II) and $1 \mathrm{~cm} * 0.5 \mathrm{~cm}$ (phase I). The patients were debrided, and dressings were changed every day. The pressure sores in the sacrococcygeal region and hip were wrapped with hydrogel foam dressing, and the heel was bandaged with sterile gauze, which was replaced every other day. The heel wound had mostly healed in 6 days, the hip had mostly healed in 2 weeks, and fresh granulation epithelial tissue had grown in the sacrococcygeal region, covering $60 \%$, when the patient was discharged from the hospital. No pressure ulcer was found during hospitalization.

\section{Psychological support}

diabetic patients are prone to some adverse psychological reactions, such as anxiety and depression, due to long-term fingertip blood glucose monitoring, insulin injection and complications. In addition, infection with NCIP causes isolation during hospitalization; family members are unable to visit, and the death rate is high, which causes patients to be pessimistic and fearful. Therefore, it is very important to pay longterm attention to the psychological state of patients and to give patients additional care and encouragement. For example, a patient suffered from NCIP infection after his wife and daughter had the 
disease, and his illness developed rapidly and became serious. He had 3 pressure ulcers. He was somewhat hostile to the medical staff and was not very cooperative with invasive operations such as blood glucose measurement. After we learned of these conditions, we increased communication with him, enhanced the emotional exchange, asked him as many questions as possible, introduced him to cured patients, helped him to eliminate fear, and increased basic care, especially pressure ulcer care. After 6 days, the patient's condition was controlled, the wound surfaces of pressure sores were mostly healed, and all nursing operations were well coordinated.

\section{Pharmaceutical care}

Nursing staff should be familiar with the indications and adverse reactions of various antiviral, antibacterial and hormone drugs. The drugs should be given on time and accurately according to the doctor's advice while closely monitoring for adverse reactions. Patients receiving drugs such as oseltamivir should be mainly monitored for nausea, vomiting, diarrhoea, abdominal pain, bronchitis, and cough. Adverse reactions to antibacterial drugs mainly include gastrointestinal symptoms, dysbacteriosis, and renal function damage. During the use of glucocorticoids, medium-acting or longacting insulin can be used to control blood sugar. However, monitoring of blood sugar should be performed, and insulin use should be adjusted according to the results. Adverse reactions to glucocorticoids mainly include secondary infection, blood sugar elevation and femoral head necrosis. During the SARS outbreak, patients treated with large doses of hormone were warned regarding the occurrence of posterior femoral head ischaemic necrosis within a few months after discharge from the hospital. One patient experienced vomiting twice during treatment, but no symptoms such as diarrhoea, renal function damage, and gastrointestinal haemorrhage occurred.

\section{Discharge guidance}

The isolation can be stopped if the patient has negative detections of respiratory pathogenic nucleic acids for two consecutive times (sampling time interval of at least $1 \mathrm{~d}$ ). When the patient reaches the discharge standard, the healthy family members should be informed to undergo the discharge formalities. Protective isolation measures should also be taken for discharged patients. In case of fever or repeated symptoms, timely follow-up visits should be conducted.

When discharged from the hospital, the treatment plan should be explained to patients and their families, insulin injection methods should be taught, information on relevant medical equipment and consumables should be provided (e.g., insulin pen, portable blood glucose meter), and diabetes education on hyperglycaemia identification and blood glucose monitoring should be provided. A follow-up visit should be scheduled with an endocrinologist at 1 month after discharge from the hospital [18].

Post-discharge recommendation: Reasonable diet: A healthy weight should be maintained, and fewer greasy foods and a reasonable diet including fruits should be eaten; Appropriate sports: Moderate and low-intensity sports lasting approximately 150 minutes a week, such as marking time, Taijiquan, and aerobics, should be performed. Treatment according to instructions: Hypoglycaemic drugs or insulin injections should be used according to the doctor's advice regarding time and quantity. Blood sugar 
monitoring: For patients taking oral medicine, it is recommended to monitor fasting blood sugar 2-4 times a week or 2 hours after meals. For those who use basic insulin, it is recommended to monitor fasting blood glucose during the morning. For pre-mixed insulin therapy, it is recommended to monitor fasting blood glucose in the morning and before dinner. Hygiene: Masks should be worn, and hands should be washed frequently. Symptoms of 2019-nCoV infection and acute complications of diabetes should be reported. Comprehensive control: Daily self-monitoring of blood pressure and regular blood lipid profile detection should be performed in appropriate patients [19]. Mental health: People should work and rest regularly, keep a pleasant mood, and correct their mentality in response to the epidemic. We should attach importance to the epidemic without panic.

\section{Conclusions}

When treating diabetes patients complicated with NCIP, in addition to considering the characteristics of NCIP as an infectious disease, it is also necessary to pay attention to the influence of NCIP on the blood sugar of patients. Patients should be assisted in adjusting their diet, medication, and blood sugar monitoring in a timely manner to control their blood sugar. The nutritional status of patients should be improved to enhance their resistance, prevent infection, and provide a foundation for the final cure of NCIP.

Pneumonia caused by 2019-nCoV is highly infectious, with many modes of transmission and many infected and suspected infected people. To effectively reduce 2019-nCoV infection specifically in diabetic patients, it is necessary to organize, control and manage the ward environment, work flow, medical personnel, nursing staff, family members, visitors and other aspects to ensure that patients are not infected and reduce the occurrence of nosocomial infection.

The battle against the NCIP has already begun. We will face major challenges in this sudden outbreak, face up to the danger without fear, unite as one, overcome the difficulties together and strike at the disease directly. It is hoped that by strengthening diabetes management in the context of prevention and control of new coronavirus, we can contribute to the control of the epidemic and win victory in this battlefield without smoke.

May the severe cold wreak havoc and roar, and spring be safe!

\section{Abbreviations}

2019-nCoV:2019 novel coronavirus;NCIP:novel coronavirus-infected pneumonia; SARS: respiratory distress syndrome; ICU:intensive care unit; MERS-CoV:Middle East Respiratory Syndrome coronavirus

\section{Declarations}

\section{Ethical approval and consent to participate}


Ethical approval was obtained from the ethics review committees at the First Hospital of Lanzhou University (Lanzhou, China) prior to the survey launch. Inform consent was obtained from all the patients by e-signing the inform consent online.

\section{Consent for publication}

"Not applicable" in this section

\section{Availability of data and materials}

"Not applicable" in this section

\section{Competing interests}

All other authors report that there are no financial relationships with commercial interests.

\section{Funding}

"Not applicable" in this section

\section{Authors' contributions}

MLH and CJG drafted this manuscript. ZT and LJ collected the data.ZKP helped with data analysis. All people have read, reviewed, and approved this manuscript. All authors contributed to the analysis, interpretation of results and drafting the critical review of the manuscript. All authors reviewed and approved the final version of the manuscript.

\section{Acknowledgments}

The authors thanks Fangcang Hospital. Thanks to all those who contributed to this study, especially the patients.

\section{Author details}

${ }^{1}$ The first hospital of Lanzhou University, Gansu Province, PR China; ${ }^{2}$ Department of Spine Surgery, The 940 Hospital of joint Logistics Support force of Chinese People's Liberation Army; ${ }^{*}$ Corresponding author

\section{References}

1. Huang C, Wang Y, Li X, et al. Clinical features of patients infected with 2019 novel coronavirus in Wuhan, China. The Lancet. 2020;395(10223):497-506. DOl:https://doi.org/10.1016/S01406736(20)30183-5

2. Clinical Features and Short-term Outcomes of 144 Patients With SARS in theGreater Toronto Area. JAMA. 2003;289(21):2801. doi:10.1001/jama.289.21.J0C30885 
3. AllardR, Leclerc P, Tremblay C, Tannenbaum T-N. Diabetes and the Severity of Pandemiclnfluenza A (H1N1) Infection. Diabetes Care. 2010;33(7):1491-1493.doi:10.2337/dc09-2215

4. vanden Brand JM, Smits SL, Haagmans BL. Pathogenesis of Middle East respiratorysyndrome coronavirus: MERS coronavirus infection in humans and animals. JPathol. 2015;235(2):175-184. doi:10.1002/path.4458

5. Yang J-K, Lin S-S, Ji X-J, Guo L-M. Binding of SARS coronavirus to its receptor damages islets and causes acute diabetes. Acta Diabetol. 2010; 47(3):193-199.doi:10.1007/s00592 -009-0109-4

6. Wu F, Zhao S, Yu B, et al. A new coronavirus associated with human respiratory disease in China. Nature. 2020 doi:10.1038/s41586-020-2008-3

7. Read JM, Bridgen JRE, Cummings DAT, et al. Novel coronavirus 2019-nCoV: early estimation of epidemiological parameters and epidemic predictions[J]. medRxiv (preprint), 2020. DOI: https://doi.org/10.1016/S0140-6736(20)30183-5.

8. Tang B, Wang X, Li Q, et al. Estimation of the Transmission Risk of 2019-nCov and Its Implication for Public Health Interventions[J]. J Clin Med.2020 DOI:10.3390/jcm9020462

9. Shen M, Peng Z, Xiao Y, et al. Modelling the epidemic trend of the 2019 novel coronavirus outbreak in China[J]. medRxiv (preprint), 2020. DOI: https://doi.org/10.1101/2020.01.23.916726.

10. Liu T, Hu J, Kang M, et al. Transmission dynamics of 2019 novel coronavirus (2019-nCoV)[J]. bioRxiv (preprint), 2020. DOl: https://doi.org/10.1101/2020.01.25.919787.:

https://doi.org/10.1101/2020.01.25.919787.

11. Seto WH, Tsang D, Yung RW, et al. Effective of precautions against droplets and contact in prevention of nosocomial transmission of severe acute respiratory syndrome (SARS). Lancet, 2003, 361(9368):1519-1520.

12. Medical administration and hospital authority. Notice of the General Office of the National Health and Health Commission on Issuing Guidelines on the Scope of Use of Common Medical Protective Equipment in Prevention and Control of Pneumonia Infected by New Coronavirus (Trial) [EB/OL]. (2020-01-27) [Access on 2020-01-31].

http://www.nhc.gov.cn/yzygj/s7659/202001/e71c5de925a64eafbe1ce790debab5c6.shtml.

13. Markwell H, Godkin D. Visitor restrictions during a public health emergency: ethical issues and guidelines for policy development. Ministry of Health and Long Term Care,2004.

14. Currie K, Curran E, Strachan E, et al. Temporary suspension of visiting during norovirus outbreaks in NHS Boards and the independent care home sector in Scotland: a cross-sectional survey of practice. J Hosp Infect, 2016,92(3):253-258.

15. Yin Hong, Wang Aimin. Exploring the visiting system for preventing the input and transmission of SARS, Shanghai Nursing, 2004, 4(2):49-50.

16. McCleary L, Munro M, Jackson $L$, et al. Impact of SARS visiting restrictions on relatives of long-term care residents. Journal of Social Work in Long-Term Care, 2006,3(3-4):3-20.

17. Li Sheyu, Huang Wenzhi, Li Wenmin, et al. Disease control of 2019 novel coronavirus infection in hospital: West China urgent recommendation. Chinese Journal of Evidence-Based Medicine, 
2020,20(2):125-132.

18. The endocrinologist branch of the Chinese physicians association, the Chinese inpatient blood glucose management expert group. consensus of Chinese inpatient blood glucose management experts [J]. Chinese journal of endocrinology and metabolism, 2017,33(1):1-10. DOI:10.3760/cma.j. issn.1000-6699.2017.01.001.

19. Diabetes Credit Association of Chinese Medical Association. Guidelines for Prevention and Treatment of Type 2 Diabetes in China (2017 Edition) [J]. Chinese Journal of Diabetes, 2018,10(1):467. DOI:10.3760/cma.j. issn.1674-5809.2018.01.003. 